\title{
Tecnologías para remover metales pesados presentes en aguas. Caso Cromo y Mercurio
}

\section{Technologies to remove heavy metals present in waters. Chrome and Mercury case}

\author{
R. A. Sarria-Villa* (iD), J. A. Gallo-Corredor ${ }^{(i)}$ y R. Benítez-Benítez \\ Departamento de Química, Universidad del Cauca, Colombia
}

\begin{abstract}
Resumen. La contaminación de los recursos hídricos por metales generados por las diferentes actividades humanas es un problema que viene en crecimiento. Los efectos adversos al medio ambiente y a los seres vivos crea la necesidad de seguir en la búsqueda de métodos que permitan su control y eliminación. Tecnologías de tratamiento como la precipitación química, adsorción, filtración en membrana, electrodiálisis y fotocatálisis han sido desarrolladas para remover metales pesados presentes en aguas. Este tipo de tecnologías muestran su capacidad para controlar la contaminación por metales como el cromo ( $86 \%$ ) y el mercurio $(89 \%)$, metales empleados en procesos industriales y productivos y cuyos residuos llegan a las fuentes hídricas.
\end{abstract}

Palabras Claves. Metales pesados, contaminación, tecnologías de remoción.

\begin{abstract}
The contamination of water resources by metals generated by different human activities is a problem that is growing. Adverse effects to the environment and to living beings create the need to continue in the search for methods that allow their control and elimination. Treatment technologies such as chemical precipitation, adsorption, membrane filtration, electrodialysis and photocatalysis have been developed to remove heavy metals present in water. This type of technology shows its capacity to control contamination by metals such as chromium $(86 \%)$ and mercury $(89 \%)$, metals used in industrial and productive processes and whose waste reaches water sources.
\end{abstract}

Keywords. Heavy metals, pollution, removal technologies.

Como Citar. R. A. Sarria-Villa, J.A. Gallo-Corredor y R. Benítez Benítez, "Tecnologías para remover metales pesados presentes en aguas. Caso Cromo y Mercurio", Jou. Cie. Ing., vol. 12, no. 1, pp. 94-109, 2020. doi: $10.46571 / \mathrm{JCl} .2020 .1 .8$

Recibido: 11/09/2019 Revisado: 17/04/2020 Aceptado: 11/06/2020

\section{Introducción}

La minería en Colombia constituye uno de los principales motores económicos del país. En los últimos años Colombia ha intensificado su explotación minera en razón del uso de

* e-mail:rodrigosv@unicauca.edu.co 
nuevas tecnologías y de la llegada de inversionistas extranjeros al país. Colombia cuenta con una ubicación privilegiada desde el punto de vista metalogénico debido al marco geológico que lo caracteriza, pues posee dos grandes provincias geotectónicas: el Cinturón Andino, en éstas provincias geotectónicas se presentan gran variedad de ambientes geológicos que han sido identificados en otros países con yacimientos de minerales y piedras preciosas, metales básicos, productos mineros industriales, rocas ornamentales, uranio y carbón, factores que atraen las inversiones extranjeras para realizar labores de exploración y explotación.

En Colombia la minería del oro se realiza de tres grandes tipos: La gran minería, la mediana y la artesanal o pequeña minería. La gran minería del oro está asociada generalmente con empresas multinacionales, y tanto la mediana como la pequeña con compañías o agremiaciones nacionales o locales. La pequeña y mediana minería en Colombia utiliza la amalgamación del oro como proceso para su extracción de las rocas que lo contienen. Este proceso es realizado utilizando mercurio elemental, el cual forma aleaciones con varios metales, entre ellos el oro, y de esta forma logra extraerlo del material rocoso. Una vez formada la amalgama oro-mercurio, esta es calentada y el mercurio elemental evaporado, quedando una mezcla de oro y otros metales en menor proporción.

El mercurio evaporado es inhalado directamente por los mineros en varias etapas de la extracción aurífera, especialmente durante la quema de la amalgama, por lo que en la cadena productiva, los más afectados son los quemadores de amalgamas [1]. Parte de este mercurio llega a la atmósfera, es depositado en los cuerpos de agua cercanos y transformado por las bacterias a metilmercurio, ión que se biomagnifica a través de la cadena trófica, alcanzando los peces, y eventualmente a sus consumidores, entre ellos el hombre. En Colombia, otros sectores productores afectan y deterioran el medio ambiente, siendo el sector pecuario, agrícola, doméstico e industrial, los principales sectores productores de residuos sólidos. Entre las principales industrias que generan gran cantidad de sólidos se encuentra la industria del cuero. En la producción de la industria de curtiembres se manipulan residuos sólidos, líquidos y gaseosos.

Los residuos sólidos se originan tanto en curtiduría como en los productos terminados. El proceso con mayor producción de residuos es el de ribera, seguido por el de curtido y, por último, se disminuye la concentración residual durante el proceso de terminado. El componente físico de los residuos de las curtiembres genera efectos negativos sobre los recursos hídricos, disminuyendo la presencia de oxígeno disuelto, aumentando la salinidad y repercutiendo con mayor profundidad sobre las aguas subterráneas, ya que estas carecen de aireación. En Colombia sobresalen en la industria de las curtiembres las siguientes ciudades y municipios: en Cundinamarca, Villapinzón y Bogotá; en el Valle del Cauca, Cartago y Cerrito; en Nariño, Pasto y Belén; en Tolima, Ibagué; y en Quindío, La María. Sin embargo, no hay que descartar las industrias que existen en otras zonas del país como el Valle de Aburrá, Cúcuta, Barranquilla y Cartagena; ya que, por ejemplo, desde estas tres últimas se transportan pieles crudas a Villapinzón y Bogotá para su procesamiento [2]. Las grandes metrópolis contribuyen a deteriorar los recursos hídricos mediante la afluencia de residuos de índole orgánica, por la presencia de patógenos. Entre las localidades más contaminantes están Bogotá, el Valle de Aburrá, el área Cali-Yumbo-Palmira y el área de Barranquilla.

La acumulación de residuos sólidos contribuye a las descargas de lixiviados, con altas concentraciones de residuos peligrosos como fenoles, cromo, mercurio y plomo. En consecuencia, la industria de curtiembres vierte a los ríos Bogotá, Cali, Pasto y Medellín, metales pesados altamente tóxicos como el cromo [3]. Los efectos negativos de la presencia de sustancias químicas peligrosas en los cuerpos de agua crea la necesidad de incrementar los controles de descargas por parte de las empresas y la creación y adaptación de tecnologías de depuración y limpieza de las diferentes matrices ambientales. 


\section{Actividades productivas que involucran el uso de mercurio y cromo}

La minería en pequeña escala, tradicional y de hecho ha sido reconocida por distintos instrumentos internacionales como un tipo de producción que tiene profundas raíces históricas y sociales y que está asentado en la cultura y la economía de América Latina. La minería tradicional y de hecho en Colombia, presente en el $44 \%$ de los municipios del país, y que representa el $30 \%$ del total de las explotaciones mineras, ha sido desarrollada desde la época colonial por mineros que han transmitido sus conocimientos a sus descendientes y que han encontrado en esta actividad su única forma de subsistencia; en particular, en zonas con poca presencia de la institucionalidad del Estado. No obstante, de un tiempo para acá la mecanización de esta actividad, su clandestinidad, el escaso control de las autoridades, la situación de orden público y el uso desaforado de insumos químicos como el cianuro y el mercurio (en el caso de la minería de oro) han traído nefastas consecuencias para los ecosistemas, con una agravio especial para los recursos hídricos, que ha convertido a la minería de hecho en el problema más sensible desde el punto de vista del impacto ambiental que tiene hoy en día el país.

Los mayores efectos sobre los recursos hídricos se deben principalmente a dos factores. De una parte y, en gran medida, las explotaciones carboníferas del país se realizan en zonas de páramo que inciden en las principales fuentes de abastecimiento de agua dulce y de otro lado, la fiebre del oro en varias regiones conlleva la utilización de toneladas de productos químicos que terminan en los ríos circunvecinos y en las aguas freáticas, lo cual genera problemas de salubridad pública irreversibles, con consecuencias a corto y largo plazo.

Debido a que el mercurio elemental no es absorbido fácilmente por la piel o por el tracto gastrointestinal, los efectos adversos en la salud de esta forma mercurial se generan, en mayor proporción, cuando en forma gaseosa sus vapores son absorbidos a través de los pulmones. La intoxicación aguda de mercurio elemental puede generar dificultades respiratorias, bronquitis, neumonitis intersticial y lesiones en la mucosa respiratoria. El mercurio en formas inorgánicas, distintas a la metálica, puede ser tóxico también cuando se ingiere, pues ocasiona espasmos intestinales, diarrea y vómito.

Los cuadros de toxicidad aguda por ingestión de metilmercurio se caracterizan por síntomas como parestesias, ataxia, neurastenia, espasticidad, disminución de agudeza visual, pérdida de audición y memoria, defectos de aprendizaje, falta de coordinación y debilidad muscular. En las aguas con abundante materia orgánica, en particular donde hay buena sedimentación, el mercurio metálico se convierte en metilmercurio, el cual, a través de la cadena trófica (microorganismos invertebrados acuáticos - peces), se acumula en los peces grandes, sobre todo en los carnívoros. Son precisamente estos últimos los que presentan mayor contaminación mercurial y los de mayor consumo por parte del hombre, que está en la cima de la cadena alimenticia.

Finalmente, cabe resaltar que, según un informe de las Naciones Unidas, Colombia es el lugar del mundo con mayor contaminación de mercurio en el aire y en el agua, teniendo en cuenta que cada año se eliminan al ambiente, en promedio, entre 50 y 100 toneladas de mercurio durante el proceso de extracción del oro, por lo cual el país ocupa el deshonroso lugar del mayor contaminador con mercurio per cápita del mundo. En los pueblos de Antioquia: Remedios, Segovia y Zaragoza se han encontrado serios problemas de salud relacionados con la contaminación con mercurio.

En un estudio realizado por las Naciones Unidas se estableció que los niveles de mercurio en algunas áreas urbanas de Remedios, Segovia y Zaragoza pueden llegar a ser 1000 veces más altos que los aceptados por la Organización Mundial de la Salud. Cerca de 15 trasplantes de riñón se llevan a cabo cada año en Remedios, debido a la contaminación con mercurio en el aire y en el agua. De igual forma, en Segovia y Remedios, se ha detectado en niños trastornos como déficit atencional y problemas de memoria y de lenguaje. En gran cantidad de minas de oro del departamento del Cauca y Valle se aplica el proceso de amalgamación a cielo abierto, es decir que todo el material aurífero a tratar se pone en contacto directo con mercurio en medio acuoso. 
En el proceso de amalgamación se utilizan cerca de 112 gramos de mercurio para beneficiar 1.87 gramos de oro, cifra extremadamente alta ya que se esperan 10 gramos de mercurio por 1 gramo de oro [4].

En el Valle del Cauca, la extracción de oro se da principalmente en los municipios de Dagua, Buenaventura, Ginebra, Buga, Tuluá, Andalucía, Bugalagrande y Jamundí. Actualmente, las zonas más preocupantes de extracción de minería ilegal son la del pacifico, la cual se ve afectada por la extracción de oro, incluida la cuenca del Rio Dagua, así como Ginebra, específicamente la cuenca del Rio Guabas. Ya en extracción a menor escala, se resaltan igualmente la cuenca alta de los ríos frío y Jamundí [5]. En la figura 1 se presenta el municipio de Dagua-Valle-Colombia, donde se lleva a cabo la actividad de extracción de oro presentando gran afectación al río Dagua.

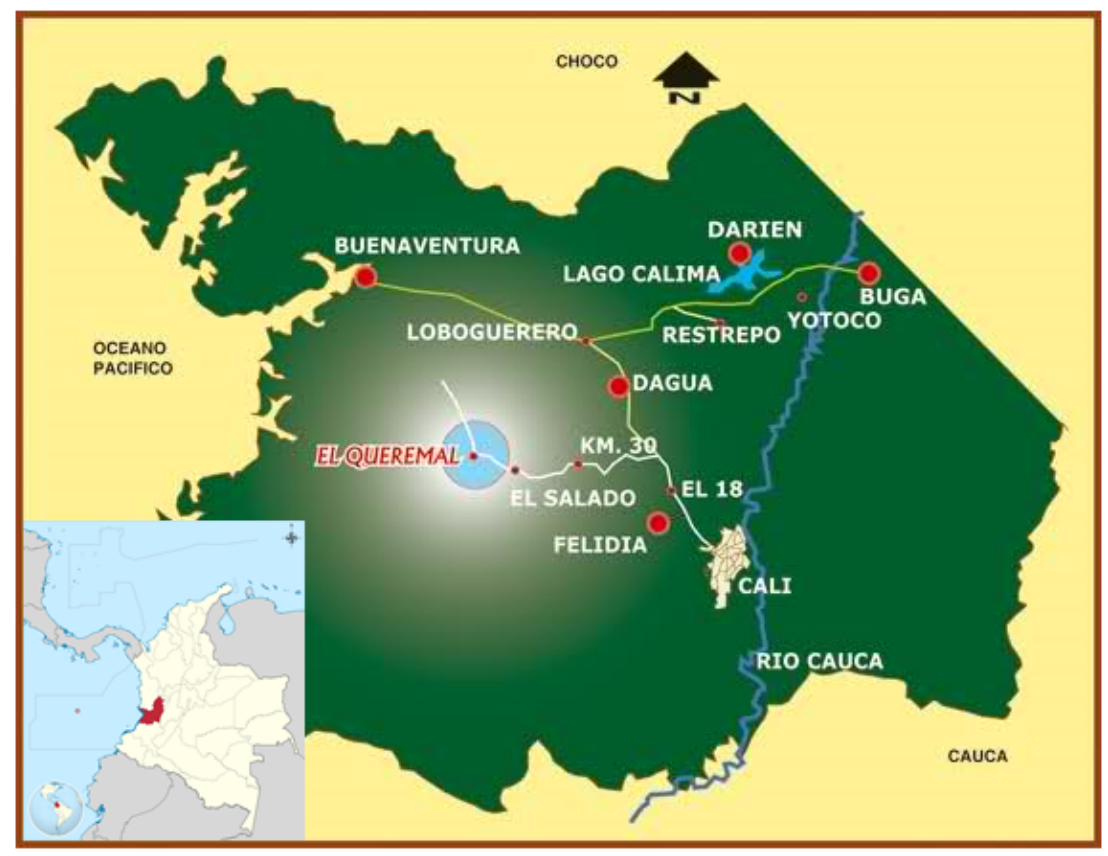

Figura 1: Zona del municipio de Dagua Valle con actividad de extracción de oro [6].

Por otro lado, el cromo que es muy utilizado en la industria de curtiembres como agente antipudrición y en otras etapas del proceso, puede encontrarse en la naturaleza como Cr III y Cr VI. El Cr III a niveles traza es esencial para la vida, mantiene el metabolismo de la glucosa, lípidos y proteínas. Sin embargo, como todos los metales a altas concentraciones es tóxico. El Cr VI es 30 veces más dañino que el Cr III, y se le atribuyen propiedades de ser cancerígeno y mutagénico por su facilidad de penetrar en membranas y alterar el ADN. El cromo se utiliza en diferentes actividades industriales como metalurgia, pinturas y curtido del cuero, en esta última se utilizan sales de Cr III, siendo un problema la posibilidad de transformación de éste en cromo hexavalente por oxidación [7].

Estudios de los niveles de mercurio y cromo en la laguna de Sonso [8], mostraron niveles moderados de mercurio en la zona de la laguna principalmente influenciada por contaminación del río Cauca por la actividad industrial de Yumbo y también influencia por la actividad de extracción de oro que se lleva a cabo en el Departamento del Valle. Este mismo estudio mostró que los niveles de cromo encontrados en el área de estudio son relativamente altos con concentraciones promedio de $75 \mathrm{mg} / \mathrm{kg}$, siendo de 75 a $100 \mathrm{mg} / \mathrm{kg}$ catalogado como una contaminación severa de los sedimentos. Los niveles de cromo están asociados con actividades industriales como galvanización, electroplateado, pinturas y aluminio. Estas actividades son 
llevadas a cabo en Cali, Palmira y Yumbo.

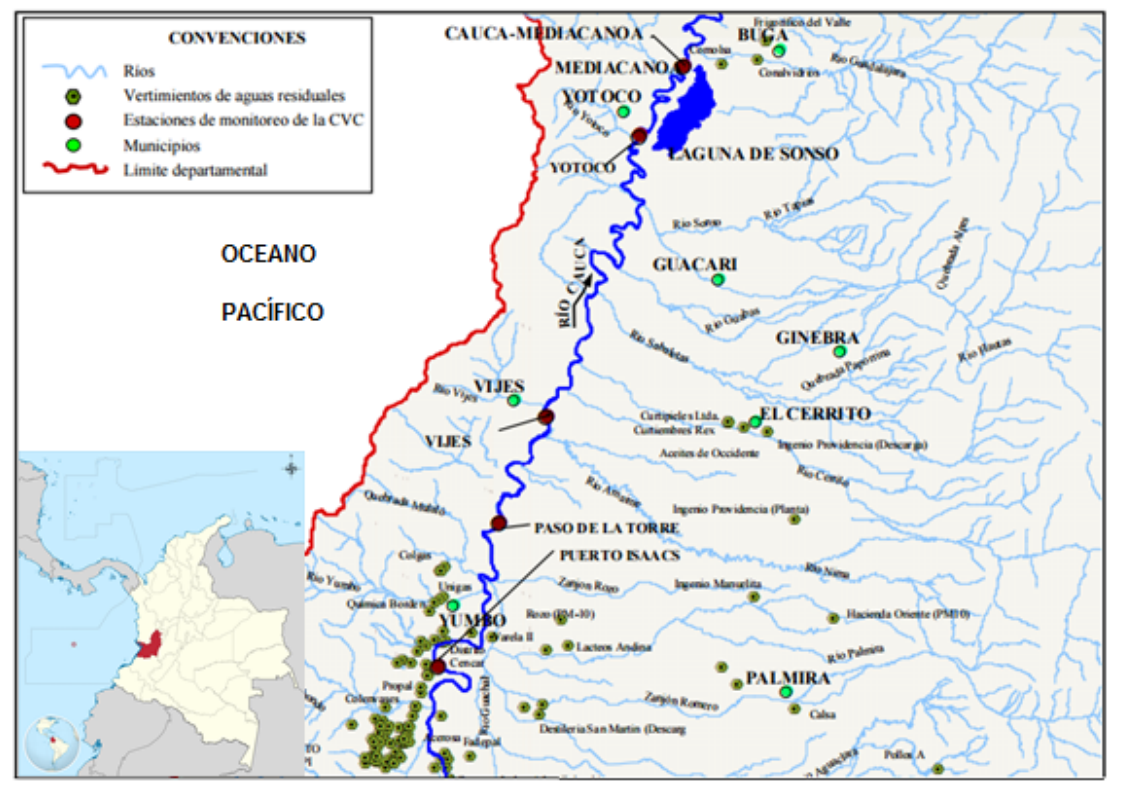

Figura 2: Zona del rio Cerrito con descargas de actividad de curtiembres [7].

En la región del norte de Cerrito, las principales fuentes de cromo son las curtiembres de cuero, las cuales descargan los residuos de cromo al río Cerrito y este a su vez descarga sus aguas en el río Cauca. Las curtiembres utilizan el óxido de cromo $\left(\mathrm{Cr}_{2} \mathrm{O}_{3}\right)$ como agente antipudrición y en otras etapas del procesamiento del cuero. El estado de oxidación del $\mathrm{Cr}_{2} \mathrm{O}_{3}$ es $3^{+}$, sin embargo, una vez la sustancia está en solución a un pH mayor de 3.5, el cromo cambia su estado de oxidación a $6^{+}$, el cual es altamente tóxico y cancerígeno. En la figura 2 se presenta el mapa de ubicación de algunas de las curtiembres que descargan sus residuos al río Cerrito en el Valle del Cauca. Estudios de composición mineralógica en minas presentes en el departamento del Cauca han arrojado resultados de un $20 \%$ en composición de minerales metálicos, presentando como minerales principales la calcopirita, pirita, esfarelita, galena, pirrotita, arsenopirita, hematita e ilmenita [9].

En una visita realizada a una de las más grandes curtiembres en 2004 por el grupo de Gischler, se supo del uso de más de una tonelada de $\mathrm{Cr}_{2} \mathrm{O}_{3}$ por día en un volumen de $450 \mathrm{~m}^{3}$ de agua [8]. En estudios realizados por la CVC se han encontrado concentraciones de $2.27 \mathrm{mg} / \mathrm{l}$ de cromo en muestras de agua del río Cerrito, 50 metros corrientes abajo de las curtiembres. El río Cerrito se conecta con el río Cauca a la altura de la laguna de Sonso en el Valle del Cauca cerca a la estación de Mediacanoa. En el trabajo realizado por Gischler con el apoyo de la CVC se encontraron concentraciones de cromo en sedimentos de $5.33 \mathrm{~g} / \mathrm{kg}$ en la desembocadura del río Cerrito al río Cauca y de mercurio de $1.20 \mathrm{mg} / \mathrm{kg}$ en la desembocadura del Canal Navarro al río Cauca [8]. En un trabajo realizado por la CVC en 2005 se encontraron concentraciones de mercurio en el Canal Navarro entre $<90-139.6 \mu \mathrm{g} / \mathrm{kg}$ y de Cromo de $36.0-73.4 \mathrm{mg} / \mathrm{kg}$ [10]. Metales como el cromo y el mercurio se encuentran entre los metales más peligrosos descargados a las fuentes de agua por las diferentes industrias químicas. Debido a su alta solubilidad en ambientes acuáticos, los metales pesados pueden ser absorbidos por los organismos vivos. Una vez que entran en la cadena alimenticia, grandes concentraciones de metales pesados se pueden acumular en el cuerpo humano. Por lo tanto, es necesario tratar las aguas residuales contaminadas antes de ser descargadas al medio ambiente [11]. En la tabla 3, se presentan los límites permisibles de cromo y mercurio en agua y sedimentos establecidos por la USEPA [12]. 


\begin{tabular}{|l|l|l|l|}
\hline Metal Pesado & Toxicidad & $\begin{array}{l}\text { L.P } \\
(\mathbf{m g} / \mathbf{L})\end{array}$ & $\begin{array}{l}\text { Aguas Sedimentos } \\
(\mathrm{mg} / \mathrm{kg})\end{array}$ \\
\hline Cromo & $\begin{array}{l}\text { Dolor de cabeza, dia- } \\
\text { rrea, nauseas, vómito, } \\
\text { cancerígeno }\end{array}$ & 0.05 & 43.4 \\
\hline Mercurio & $\begin{array}{l}\text { Artritis reumatoide, } \\
\text { daños a los riñones, } \\
\text { sistema circulatorio y } \\
\text { sistema nervioso }\end{array}$ & 0.00003 & 0.18 \\
\hline
\end{tabular}

Tabla 1: Límites permisibles (L.P) de cromo y mercurio en aguas $(m g / l)$ y sedimentos $(m g / K g)$ [12].

Actividades industriales y de minería siguen arrojando metales pesados a las fuentes hídricas contaminando este importante recurso sin controlar de alguna manera su descarga. Los metales pesados son no biodegradables y tienden a acumularse en los organismos vivos causando varias enfermedades y desordenes. Procesos de tratamiento de corrientes contaminadas con residuos de metales incluyen precipitación química, filtración por membrana, intercambio iónico, adsorción en carbón y coprecipitación/adsorción [13]. Si se tienen en cuenta los efectos que tienen los metales pesados, debido a su presencia en el agua, es evidente que se hace necesaria su eliminación. Las industrias se ven obligadas a reducir la cantidad y la peligrosidad de sus vertidos al medio ambiente, a causa de las presiones legales, sociales y económicas. Este hecho ha conducido a que dichas industrias concentren sus esfuerzos en evacuar vertidos más limpios y en reciclar o reutilizar su contenido en proporciones significativas.

\section{Tecnologías de remoción de metales presentes en aguas residuales}

Los efluentes industriales se han tratado tradicionalmente mediante una combinación de procesos físico-químicos, como la floculación, precipitación y filtración y procesos biológicos como el de lodos activados [14]. Sin embargo, estos procesos fallan en que no consiguen reducir la concentración de los metales pesados, en unos límites inferiores a los permitidos por la legislación. Además estas tecnologías, no consiguen proveer una diferenciación clara en su composición, con lo cual se hace difícil reciclar o reusar los subproductos, y como consecuencia los lodos suponen un problema añadido para su deposición final. Las plantas de tratamiento de aguas residuales no están diseñadas para manejar residuos tóxicos. Los metales y su toxicidad persisten incluso en los lodos después del tratamiento, por esto se deben eliminar en la fuente emisora, en un pretratamiento especial diseñado a tal efecto. Este tratamiento específico no debe tener un alto coste, ya que a menudo se enfrentaría con grandes volúmenes de efluente [14]. Algunas de las tecnologías utilizadas en la separación de metales de los efluentes líquidos, para su posterior reutilización son la precipitación química, la recuperación con disolvente, técnicas de adsorción y de intercambio iónico y procesos basados en tecnología de membrana. A continuación se describen brevemente:

\subsection{Precipitación química}

La precipitación química se considera el tratamiento estándar para la eliminación de metales de todo tipo de aguas. La capacidad de eliminación de los metales de la disolución depende principalmente de dos factores: La solubilidad de la especie más soluble del metal que puede formarse y La separación del sólido de la disolución acuosa. Estos factores hacen que la precipitación sea poco efectiva cuando el metal se encuentra en concentraciones muy bajas, ya que se necesita un exceso de agente precipitante para llegar a formar un precipitado, y en muchos casos la partícula sólida formada no tiene estabilidad suficiente para separarse de la 
disolución. La precipitación química es el método más ampliamente usado para remover metales pesados de efluentes inorgánicos. El mecanismo conceptual de remoción de metales pesados por precipitación química se presenta en la ecuación 1 [15].

$$
M^{2+}+2(\mathrm{OH})^{-} \leftrightarrow M(O H)_{2} \downarrow
$$

Donde $\mathrm{M}^{2+}$ y $\mathrm{OH}^{-}$representan los iones del metal disuelto y el precipitante respectivamente, mientras que $\mathrm{M}(\mathrm{OH})_{2}$ es el hidróxido del metal insoluble. El ajuste del $p H$ a condiciones básicas ( $p H$ de 9 a 11) es el parámetro que mejora significativamente la remoción del metal por precipitación. La precipitación química tiene ventajas como el tratamiento de efluentes con altas concentraciones de metales, simplicidad en el proceso, equipo poco costoso y operación segura y conveniente. Sin embargo la precipitación química requiere gran cantidad de químicos, produce gran cantidad de lodos que deben ser tratados, precipitación lenta, agregación de metales a los precipitados e impactos ambientales negativos a largo plazo por la disposición de lodos [16].

\subsection{Recuperación con solvente}

La recuperación con solvente es una de las tecnologías de separación más establecidas para la eliminación de metales contaminantes en efluentes industriales [17]. Este proceso se utiliza sobre todo, en procesos a gran escala, donde las concentraciones del contaminante son elevadas. La tecnología permite la separación en distintas fases, los metales a recuperar se concentran en las diferentes fracciones. Cada fracción, individualmente, puede ser tratada o eliminada en una forma más eficaz en función del costo. La extracción por solventes es uno de los procesos más efectivos y económicos para purificar, concentrar y separar los metales valiosos que se encuentran en las soluciones enriquecidas, provenientes del proceso de lixiviación. Esta tecnología requiere el empleo de grandes volúmenes de agentes orgánicos extractantes, lo que hace incrementar los costos de operación.

\subsection{La tecnología de intercambio iónico}

En la tecnología de intercambio iónico se presenta un mecanismo de interacción electrostática, debido a las fuerzas de atracción de coulomb que tienen lugar cuando un ión de una disolución se intercambia por otro ión de igual signo que se encuentra unido a una partícula sólida inmóvil. Cuando las concentraciones de metal en los efluentes son bajas, el uso de membranas o la recuperación con disolvente, pierden sus ventajas y no son efectivos. En este caso, se requiere otra tecnología alternativa: el intercambio iónico. La desventaja de este método es que no puede manejar soluciones concentradas de metales y presencia de sustancias orgánicas y otros sólidos presentes en las aguas residuales [18].

\subsection{Adsorción}

La adsorción consiste en la migración de algunas sustancias de la fase gaseosa o líquida hacia la superficie de un sustrato sólido. El fenómeno de adsorción se da de forma natural en sistemas físicos, biológicos y químicos. Además, las operaciones de adsorción que emplean sólidos como el carbón activo y las resinas sintéticas son muy utilizadas en aplicaciones industriales y en la purificación de aguas residuales. La adsorción de contaminantes se puede realizar usando materiales orgánicos e inorgánicos. Para el proceso de adsorción es necesario tener en cuenta parámetros como tiempo de equilibrio, $p H$, efecto del medio iónico, concentración inicial del soluto, entre otros. Existen varios adsorbentes de bajo costo, derivados de los residuos de agricultura, procesos industriales, material natural o biopolímeros modificados, que han sido desarrollados recientemente y aplicados para remover metales pesados de aguas residuales. En general hay tres etapas principales en la adsorción del contaminante sobre el adsorbente: (i) Transporte del contaminante desde la solución a la superficie sólida (ii) adsorción sobre la 
superficie de la partícula (iii) Transporte con la partícula adsorbida. Aplicabilidad técnica y costo efectividad son los factores que juegan un mayor rol en la selección del adsorbente más adecuado para el tratamiento de efluentes inorgánicos. En la figura 3 se presenta el mecanismo de adsorción del ion cobre sobre óxido de titanio hidratado.

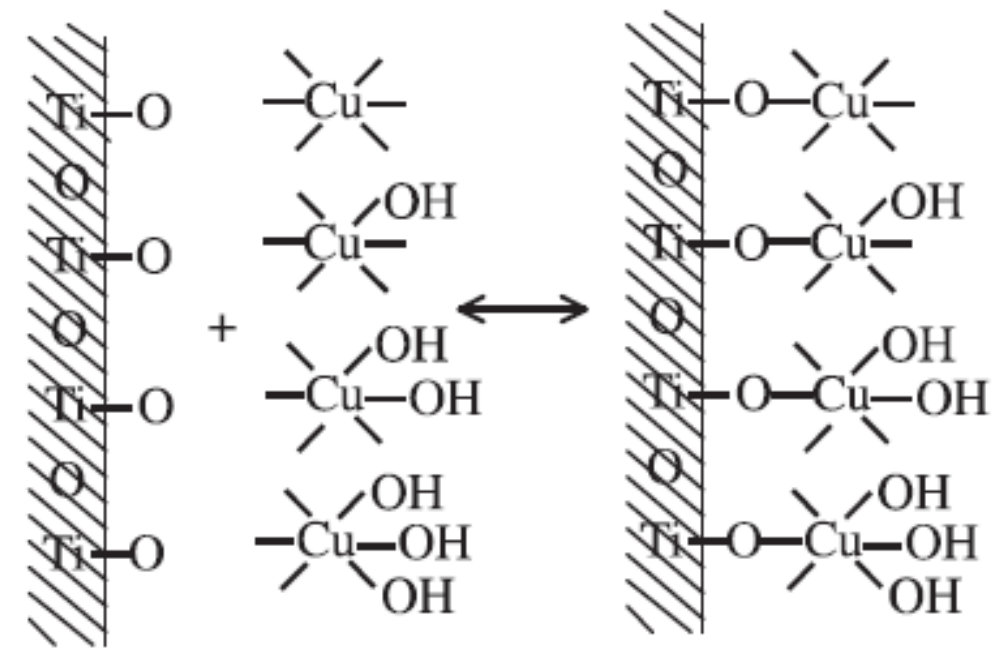

Figura 3: Mecanismo de adsorción de $\mathrm{Cu}(\mathrm{II})$ sobre óxido de titanio hidratado [19].

3.4.1. Adsorción sobre materiales naturales modificados Pretratamiento y modificación de zeolitas naturales han demostrado la mejora en la capacidad de intercambio iónico y eficiencia de remoción de metales en aguas residuales. Minerales naturales de arcilla pueden ser modificados con un material polimérico de forma que se puede mejorar significativamente su capacidad de remover metales pesados de soluciones acuosas [20].

3.4.2. Adsorción sobre sub-productos Industriales Subproductos industriales como cenizas, residuos de hierro, óxidos de titanio hidratados, pueden modificarse químicamente para aumentar la eficiencia de remoción de metales de aguas residuales [20,21] estudiaron subproductos de la industria de la fundición del hierro para la remoción de Zn(II). Cenizas volátiles fueron también investigadas como adsorbentes para remover metales tóxicos [22], exploraron cenizas de bagazo, un residuo sólido de la industria azucarera, para remover $\mathrm{Cd}(\mathrm{II})$ y $\mathrm{Ni}(\mathrm{II})$ de una solución sintética a $p H$ en un rango de 6.0 a 6.5. Aserrín tratado con fosfato de hidrógeno disódico fue usado para la adsorción de $\mathrm{Cr}(\mathrm{VI})$ a $p H 2[19,23,24]$ estudiaron el óxido de titanio anhidro para la adsorción de $\mathrm{Cr}(\mathrm{VI})$ y $\mathrm{Cu}(\mathrm{II})$ respectivamente. El uso de carbón activado obtenido a partir de corteza de pino patula para retener metales pesados en aguas industriales ha dado buenos resultados. Las pruebas de adsorción mostraron buena afinidad por el hierro, cobre y por la carga orgánica presente en el agua residual. Para el Zn no se observó buena capacidad de adsorción [25].

3.4.3. Adsorción sobre residuos agrícolas y biológicos modificados (bioadsorción) Recientemente existe un gran interés en investigaciones para remover metales pesados de efluentes industriales utilizando subproductos agrícolas como adsorbentes. El uso de subproductos agrícolas en biorremediación de iones de metales pesados, es conocido como bioadsorción. Esta utiliza biomasa microbial inactiva para enlazar y concentrar metales pesados presentes en aguas residuales por rutas puramente fisicoquímicas (principalmente quelación y adsorción) [26]. Nuevas fuentes de biomasa como cáscara de avellana, cáscara de arroz, cáscara de mazorca pueden ser usadas como adsorbentes de metales pesados después de realizar una modificación química o conversión a 
carbón activado por efecto de la temperatura. Aplicaciones con carbón de cáscara de coco modificada con agentes oxidantes y/o quitosano para remover $\mathrm{Cr}(\mathrm{VI})$ fue investigado por Babel [11,27], estudiaron la remoción de $\mathrm{Cr}(\mathrm{VI})$ presente en medio acuoso usando carbón activado de cáscara de arroz. Ellos encontraron la máxima remoción a pH 2. Cáscara de arroz, conteniendo celulosa, lignina, carbohidratos y sílice, fue investigada para la remoción de $\mathrm{Cr}(\mathrm{VI})$ en solución [28]. Para incrementar la remoción del metal, el adsorbente fue modificado con etilendiamina. La máxima adsorción de $\mathrm{Cr}(\mathrm{VI})$ fue de $23.4 \mathrm{mg} / \mathrm{g}$ a $p H$ 2. Igualmente, la corteza de eucalipto modificada químicamente se ha empleado para la remoción de mercurio en medios acuosos, obteniéndose una máxima capacidad de adsorción de $33.11 \mathrm{mg} / \mathrm{g}$ [29]. En la tabla 2 se presenta la capacidad de adsorción de metales pesados por algunos residuos agrícolas y biológicos.

\begin{tabular}{|l|l|l|l|l|l|l|l|l|}
\hline & \multicolumn{6}{|c|}{ Capacidad de adsorción (mg/g) } \\
\hline Adsorbente & $P b^{2+}$ & $C d^{2+}$ & $Z n^{2+}$ & $C u^{2+}$ & $C r^{6+}$ & $\mathrm{Ni}^{2+}$ & $\mathrm{Hg}^{2+}$ \\
\hline Cáscara de maíz & 456 & 493.7 & 495.9 & & & & \\
\hline Cáscara de naranja & & & & & & 158 & \\
\hline Carbón de cáscara de coco & & & & & 3.65 & & \\
\hline Carbón activado de cáscara de nuez & & & 13.9 & 31.7 & & & \\
\hline Cáscara de arroz & & 2.0 & & & 0.79 & & \\
\hline Cáscara de arroz modificada & & & & & 23.4 & & \\
\hline Cáscara de arroz modificada & & & & & 23.4 & & \\
\hline Spirogiyra (Alga verde) & & & & 133 & & & \\
\hline Euklonia máxima Alga marina & 235 & & & 90 & & & \\
\hline Ulva lactuca & & & & & 112.3 & & \\
\hline Especies de oedogonium & 145 & & & & & & \\
\hline Especies de Nostoc & 93.5 & & & & & & \\
\hline Biomasa bacterial de Bacillus & 467 & 85.3 & 418 & 381 & 39.9 & & \\
\hline Corteza de Pinus pinaster & & & & & & & 7.0 \\
\hline
\end{tabular}

Tabla 2: Capacidad de adsorción de algunos metales pesados por residuos agrícolas y biológicos $[11]$.

En Colombia existen cerca de 350,000 hectáreas de plantaciones forestales, principalmente de pino y eucalipto, y en el suroccidente del país, existen más de 20,000 hectáreas de Pinus patula y Pinus oocarpa, cultivadas con una finalidad industrial [30]. Las labores agroforestales generan una gran cantidad de residuos entre los que se encuentran la corteza, a partir de la cual se pueden crear nuevas alternativas como la obtención de extractos con un alto contenido de compuestos fenólicos [31], capaces de retener metales tóxicos generados en diferentes actividades productivas. Los polifenoles presentes en las cortezas de pino pueden actuar como intercambiadores iónicos [32], donde, los hidroxilos desprotonados del anillo B del polifenol quelatan dicationes como se muestra en la figura 4, lo que permite su uso para remover metales pesados de aguas residuales. 
<smiles>[R1]c1c(O)cc(O)c2c1O[C@H](c1ccc(O)c(O)c1)[C@H](O)C2[R1]</smiles><smiles>[R14]Oc1cc([C@H]2Oc3c([R1])c(O)cc(O)c3C([R1])[C@H]2O)ccc1O[Y17]#[I+]</smiles>

Figura 4: Reacción entre un polifenol y un metal divalente.

Se han realizado trabajos de adsorción de metales como el mercurio y el cadmio usando cortezas de Pinus pinaster modificado con formaldehido, estudiando su cinética e isotermas de adsorción y la influencia del $p H$. Los resultados mostraron buena afinidad de las cortezas modificadas por el cadmio y mercurio [33]. También se han usado taninos de cortezas modificados para remover metales como $\mathrm{Zn}^{2+}, \mathrm{Ni}^{2+}$ y $\mathrm{Cu}^{2+}$ de aguas residuales mediante el proceso de coagulación/floculación alcanzando remociones hasta del $75 \%$ del metal [34]. La corteza de Pinus radiata modificada con formaldehido ha sido utilizada para remover $\mathrm{Cu}(\mathrm{II}), \mathrm{Fe}(\mathrm{III}), \mathrm{Al}(\mathrm{III})$ y $\mathrm{Cd}(\mathrm{II})$ en aguas residuales con porcentajes de $15.6 \%, 46.9 \%, 83.7 \%$ y $3.3 \%$ respectivamente. En la figura 5 se presenta la isoterma adsorción acumulada en el tiempo de metales sobre corteza de pino modificada químicamente. Usando corteza de Pinus radiata modificada se han obtenido porcentajes de retención del $89.4 \%$ y $89.1 \%$ en aguas para mercurio y plomo respectivamente [32].

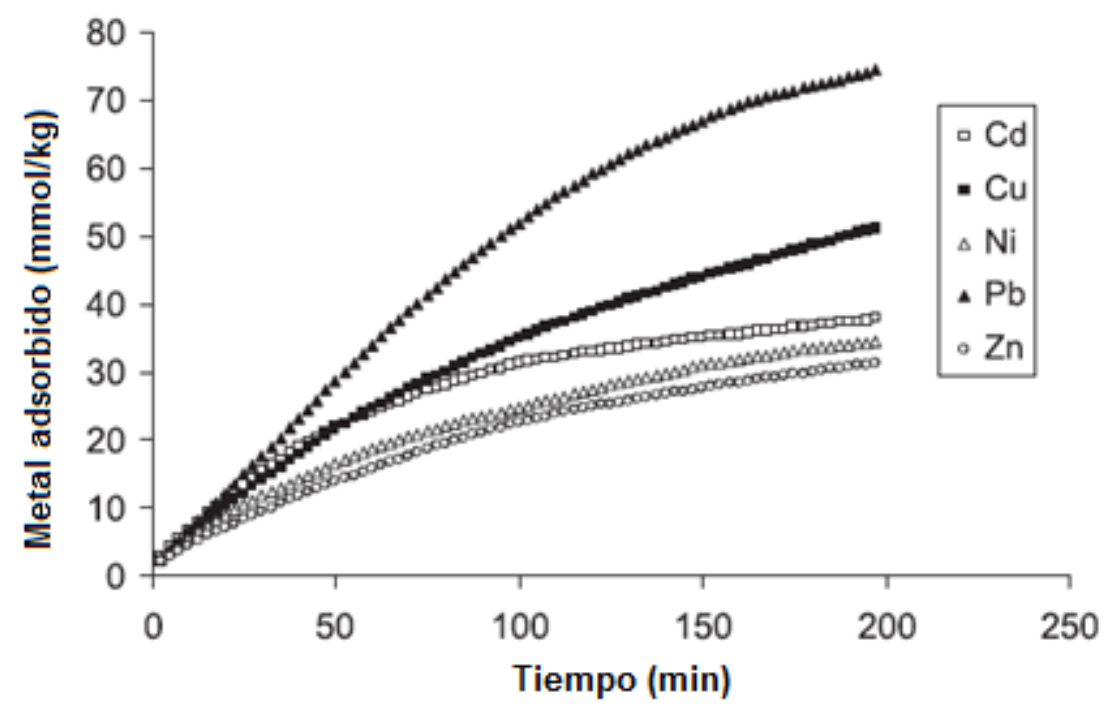

Figura 5: Isotermas de adsorción de metales sobre corteza de pino modificada [34].

3.4.4. Adsorción sobre biopolímeros modificados e hidrogeles Los biopolímeros son industrialmente atractivos porque permiten retener iones de metales de transición a bajas concentraciones [11]. Otro atractivo de los biopolímeros es que poseen un número de diferentes grupos funcionales tales como grupos hidroxilos y amino, los cuales incrementan la retención del metal y maximizan la capacidad de carga química. Se han sintetizado nuevos adsorbentes de biopolímeros a base de polisacáridos derivados de quitina, quitosano y almidón para remover metales 
pesados presentes en aguas residuales. Hidrogeles, los cuales son polímeros hidrofílicos entrecruzados, son capaces de expandir su volumen debido a su capacidad de expandirse en agua, capacidad que se emplea en la purificación de aguas residuales con presencia de metales pesados [35], sintetizaron un hidrogel de poli(etilen glicol dimetacrilato-co-acrilamida), para retener $\mathrm{Pb}(\mathrm{II}), \mathrm{Cd}(\mathrm{II})$ y $\mathrm{Hg}(\mathrm{II})$ con buenos resultados a un $p H$ superior a 6 .

\subsection{Filtración con membranas}

En la tecnología de membrana se aprovecha una diferencia de presión a través de la membrana para superar el gradiente de presión osmótica. Bajo estas condiciones, los iones no pueden atravesar la membrana y así son separados del disolvente. La filtración con membrana ha recibido considerable atención en el tratamiento de efluentes inorgánicos, debido a su capacidad de remover no solo sólidos suspendidos y compuestos orgánicos, sino también contaminantes inorgánicos tales como los metales pesados. Dependiendo del tamaño de partículas que quieran ser retenidas se pueden emplear varios tipos de membranas tales como ultrafiltración, nanofiltración y osmosis reversa para remover metales pesados presentes en aguas residuales. La ultrafiltración (UF) emplea membranas permeables para separar metales pesados, macromoléculas y sólidos suspendidos presentes en soluciones inorgánicas presentando tamaños de poro de (5-20 nm), permitiendo separar compuestos con peso molecular entre $1000 \mathrm{y}$ 100000 Da. Esta técnica permite pasar agua con solutos de bajo peso molecular, mientras retiene macromoléculas de tamaño molecular superior al del tamaño de poro de la membrana [36,37], emplearon membranas de ultrafiltración de bajo costo de $\mathrm{ZnAl}_{2} \mathrm{O}_{4}-\mathrm{TiO}_{2}$ para remover $\mathrm{Cd}$ (II) y $\mathrm{Cr}$ (III) presentes en una solución sintética con recuperaciones del $93 \%$ y $86 \%$ para Cd y Cr respectivamente. Una de las desventajas de la ultrafiltración es el taponamiento causando decaimiento del flujo que normalmente se da de 2 a 9.5 bar de presión, incremento en la presión transmembrana y biodegradación del material de la membrana [38]. Estos efectos se reflejan en los costos operacionales del sistema de membrana. En la figura 6 se observa una membrana de nanofiltración para metales pesados a base de amidoamina [39].

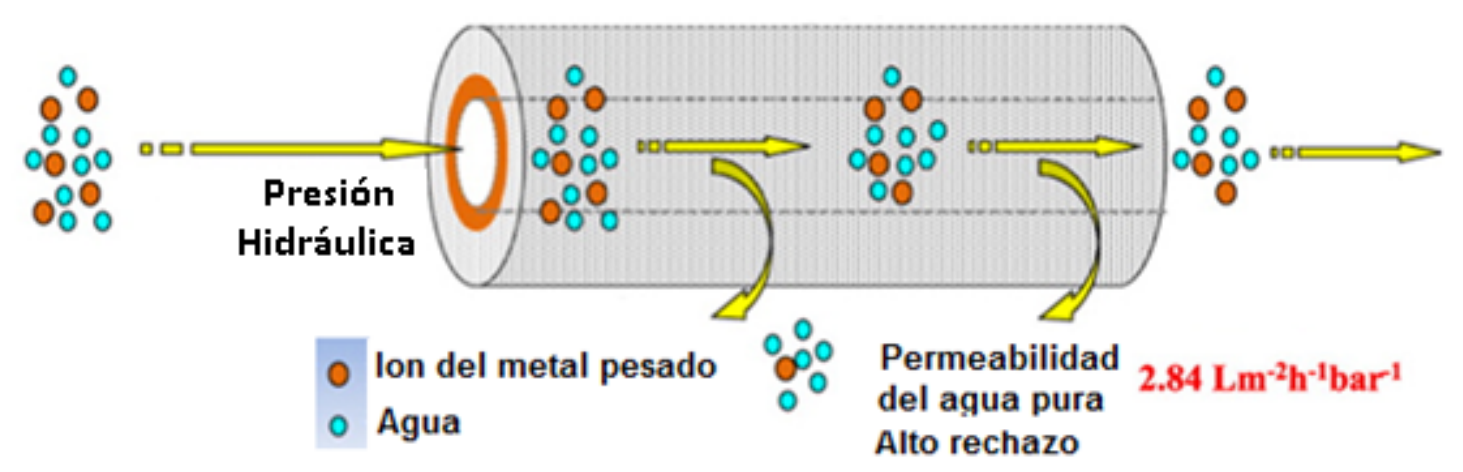

Figura 6: Membrana de nanofiltración empleada para eliminar metales en agua [40].

La aplicación de la osmosis reversa y la nanofiltración para el tratamiento de aguas residuales conteniendo los iones de cobre y cadmio fue investigada [40]. Los resultados mostraron alta eficiencia de remoción de metales por el proceso de osmosis reversa $(98 \%$ y $99 \%$ para cobre y cadmio respectivamente). La nanofiltración, permite remover el $90 \%$ de los iones de cobre presentes en los líquidos iniciales. 


\subsection{Procesos electroquímicos}

En los procesos electroquímicos se utiliza energía eléctrica como vector de descontaminación provocando reacciones de óxido-reducción tanto en el ánodo como en el cátodo. Su costo es una de las principales desventajas del proceso, sin embargo, como ventajas cabe destacar la versatilidad de los equipos, la ausencia de la utilización de reactivos y la selectividad, pues controlar el potencial del electrodo permite seleccionar la reacción electroquímica dominante deseada. En la figura 7 se presenta la remediación electroquímica de cadmio presente en lodo de agua residual [41].

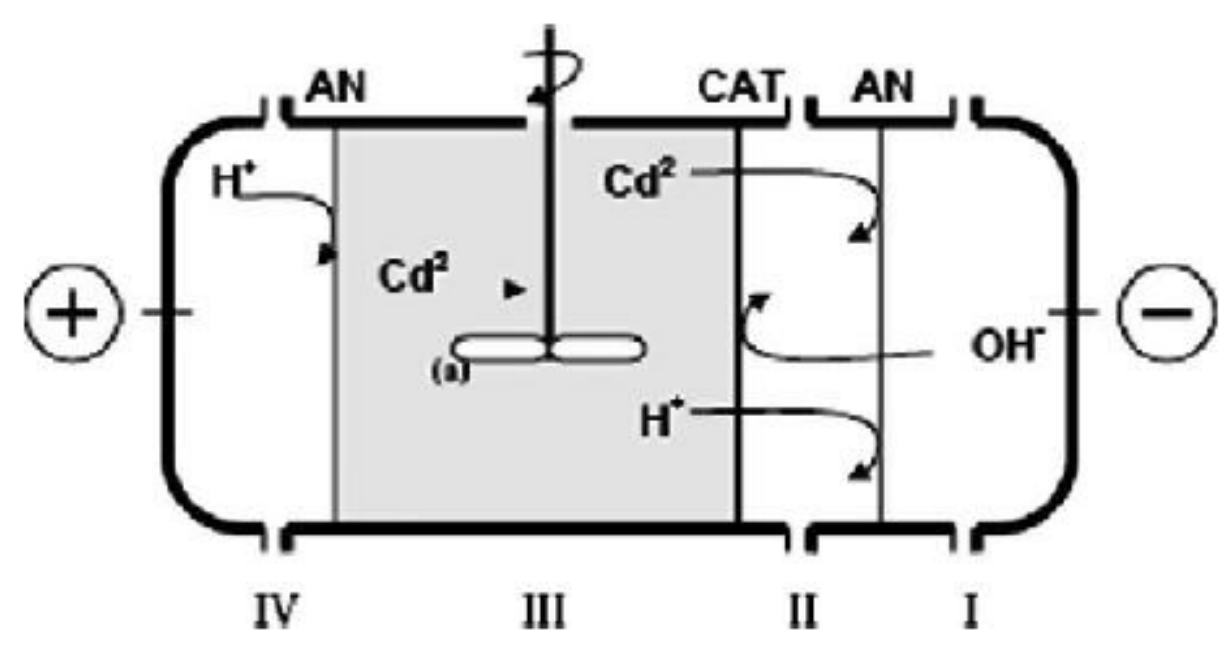

Figura 7: Remediación electroquímica de cadmio presente en lodo de aguas residuales [41].

3.6.1. Electrodiálisis En la electrodiálisis se emplea una membrana de separación en la cual las especies iónicas presentes en solución se pasan a través de una membrana de intercambio iónico por aplicación de un potencial eléctrico. Las membranas son pequeñas hojas de material plástico con alguna característica aniónica o catiónica. La electrodiálisis ha presentado eficiencias para $\mathrm{Co}(\mathrm{II})$ y $\mathrm{Ni}(\mathrm{II})$ del $90 \%$ y $69 \%$ respectivamente con concentraciones iniciales de 0.84 y 11.72 $\mathrm{mg} / \mathrm{l}$ respectivamente. Se ha observado que un incremento de voltaje y temperatura mejoran el funcionamiento de la celda, sin embargo, los porcentajes de separación decrecen con el incremento de la velocidad de flujo [42]. La electrodiálisis ofrece ventajas para el tratamiento de aguas residuales cargadas con metales pesados con la habilidad de producir una corriente altamente concentrada para la recuperación de metales y rechazo de impurezas indeseables presentes en el agua. Sin embargo, procesos de electrodiálisis con el uso de membranas requiere limpieza, cuidado en la operación y mantenimiento periódico para prevenir daños por acumulación.

\subsection{Tratamientos biológicos}

Los tratamientos biológicos constituyen una serie de importantes procesos de tratamiento que tienen en común la utilización de microorganismos (entre las que destacan las bacterias) para llevar acabo la eliminación de componentes indeseables del agua, aprovechando la actividad metabólica de los mismos sobre esos componentes. De acuerdo al elemento aceptor de electrones en el metabolismo bacteriano el sistema puede ser anaerobio, aerobio o anóxico [43].

\subsection{Fotocatálisis}

En años recientes, los procesos fotocatalíticos en suspensiones acuosas en presencia de semiconductores han recibido una considerable atención en vista de la conversión de la energía 
solar. Estos procesos fotocatalíticos han permitido una rápida y eficiente destrucción de contaminantes ambientales. Una elevada iluminación de la interface del electrolito semiconductor con una energía lumínica más alta que el band gap del semiconductor hace que pares electrónhuecos $\left(e^{-} / h^{+}\right)$sean formados en la banda de conducción y valencia del semiconductor respectivamente [44]. Estos portadores de cargas, los cuales migran a la superficie del conductor, son capaces de reducir u oxidar especies presentes en solución teniendo un adecuado potencial redox. Varios semiconductores han sido usados: $\mathrm{TiO}_{2}, \mathrm{ZnO}, \mathrm{CeO}_{2}, \mathrm{CdS}, \mathrm{ZnS}$, etc. Como generalmente se observa, las mejores eficiencias fotocatalíticas son siempre obtenidas con óxido de titanio. En la figura 8 se presenta la ruta química del proceso de fotocatálisis sobre óxido de titanio.

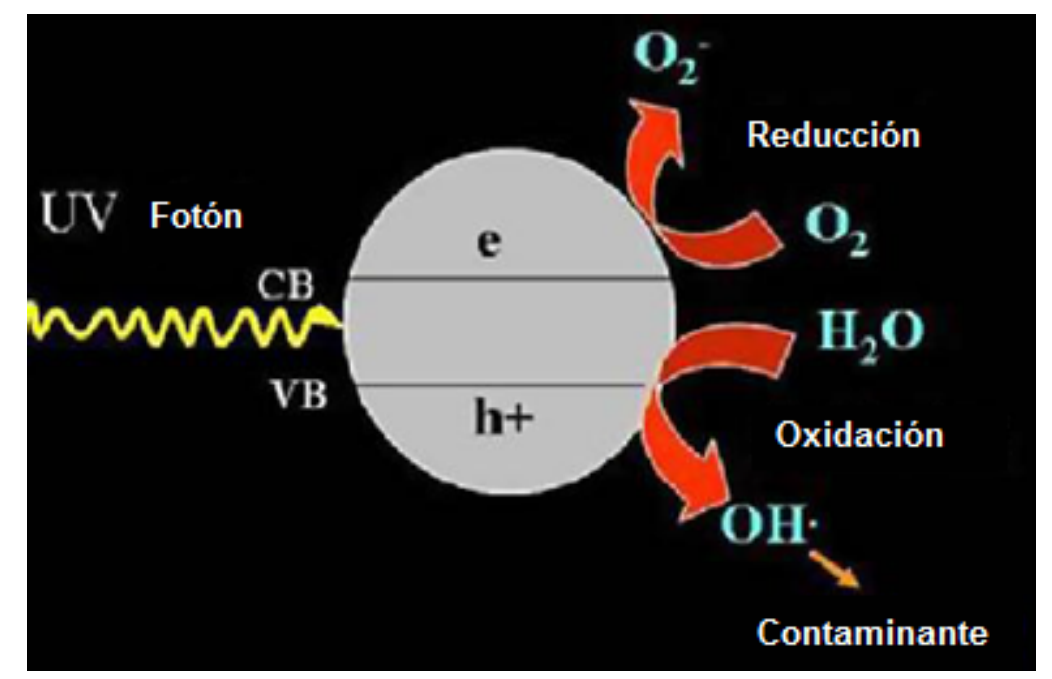

Figura 8: Ruta química del proceso fotocatalítico sobre óxido de titanio [44].

Estudios realizados por Barakat el at., 2004 [20] mostraron la remoción de cobre usando degradación fotocatalítica irradiando una suspensión de $\mathrm{TiO}_{2}$ para destruir el complejo CobreCianuro. Varios estudios también han reportado la reducción fotocatalítica de $\mathrm{Cr}$ (VI) a $\mathrm{Cr}$ (III), el cual es menos móvil y menos peligroso. Muestras preparadas a $p H$ bajo exhibieron mayor área superficial y mayor reactividad que aquellos preparados a $p H$ alto [45]. Este tipo de experimentos resultaron exitosos en un rango de concentración de cromo de 5 a 100 ppm para lograr una completa reducción de $\mathrm{Cr}(\mathrm{VI})$ a $\mathrm{Cr}(\mathrm{III})$ [46].

\subsection{Evaluación del proceso de remoción de metales pesados}

Los tratamientos fisicoquímicos para remoción de metales pesados tienen varias ventajas tales como un rápido proceso, fácil operación y control, y flexibilidad por cambio de temperatura. A diferencia de los sistemas biológicos, los tratamientos fisicoquímicos pueden manejar variables como cargas de entrada y flujos, tales como flujos temporales y descargas complejas. Sin embargo, si esto es requerido, las plantas químicas pueden ser modificadas. En adición, los sistemas de tratamiento requieren poco espacio y bajo costo de instalación. Sus beneficios, son compensados por un número de inconvenientes como el alto costo de operación debido al uso de químicos, alto consumo de energía y costo del manejo de disposición de sólidos. Sin embargo, con la reducción de costos por el uso de químicos por ejemplo utilizando adsorbentes de bajo costo y un adecuado manejo de disposición de lodos, los tratamientos fisicoquímicos vienen siendo considerados uno de los más adecuados tratamientos para efluentes inorgánicos [38]. En sistemas de aguas residuales que contienen metales pesados con otros contaminantes orgánicos, la presencia de unas 
especies usualmente impide la remoción de otros. Los más prometedores métodos para tratar sistemas complejos son los fotocatalíticos, donde se induce tanto la degradación de contaminantes orgánicos y recuperación de metales en un solo sistema, operable a cantidades traza de los compuestos de interés (menores de las ppm). La tabla 3 resume las principales ventajas y desventajas de diferentes tratamientos fisicoquímicos para metales presentes en aguas.

\begin{tabular}{|c|c|c|c|}
\hline Método de tratamiento & Ventajas & Desventajas & Referencias \\
\hline Precipitación química & Bajo costo, simple operación & $\begin{array}{l}\text { Generación de lodo, Costo ex- } \\
\text { tra de operación de disposición } \\
\text { de lodos }\end{array}$ & {$[38]$} \\
\hline $\begin{array}{l}\text { Adsorción con nuevos ad- } \\
\text { sorbentes }\end{array}$ & $\begin{array}{l}\text { Bajo costo, Condiciones de } \\
\text { operación sencillas, Amplio } \\
\text { rango de } p H \text {, Alta capacidad } \\
\text { de enlazar metales }\end{array}$ & $\begin{array}{l}\text { Baja selectividad, producción } \\
\text { de residuos }\end{array}$ & {$[12,47]$} \\
\hline Membranas de filtración & $\begin{array}{l}\text { Requerimiento de poco espa- } \\
\text { cio, baja presión, alta selecti- } \\
\text { vidad en la separación }\end{array}$ & $\begin{array}{l}\text { Alto costo de operación debido } \\
\text { al taponamiento de las mem- } \\
\text { branas. }\end{array}$ & {$[38]$} \\
\hline Electrodiálisis & $\begin{array}{l}\text { Alta selectividad en la separa- } \\
\text { ción }\end{array}$ & $\begin{array}{l}\text { Altos costos de operación de- } \\
\text { bido al taponamiento de mem- } \\
\text { branas y consumo de energía }\end{array}$ & {$[48,49]$} \\
\hline Fotocatálisis & $\begin{array}{l}\text { Remoción de metales y } \\
\text { contaminantes orgánicos si- } \\
\text { multáneamente, Menor peligro } \\
\text { por productos }\end{array}$ & $\begin{array}{l}\text { Largo tiempo de duración, } \\
\text { aplicaciones limitadas }\end{array}$ & {$[20,45]$} \\
\hline
\end{tabular}

Tabla 3: Límites permisibles (L.P) de cromo y mercurio en aguas $(m g / l)$ y sedimentos $(m g / K g)$ $[12]$.

\section{Conclusiones}

Sobre las pasadas dos décadas, las regulaciones ambientales se han vuelto más rigurosas, requiriendo una mejora de la calidad del efluente tratado. En los años recientes, un amplio rango de tecnologías de tratamientos tales como la precipitación química, adsorción, filtración en membrana, electrodiálisis y fotocatálisis han sido desarrolladas para remover metales pesados presentes en aguas residuales.

El uso de nuevos adsorbentes y membranas de filtración son los más ampliamente estudiados y ampliamente aplicados para el tratamiento de aguas residuales contaminadas con metales pesados. La fotocatálisis es una innovadora y prometedora técnica para un limpio y eficiente tratamiento.

Las tecnologías propuestas han demostrado importantes porcentajes de remoción para metales como el mercurio y el cromo. Es necesario contar con alternativas tecnológicas rentables o adsorbentes para el tratamiento de aguas residuales contaminadas con metales.

Los materiales naturales están disponibles en grandes cantidades o se presentan como residuos en actividades industriales u operaciones agrícolas, teniendo un gran potencial como adsorbentes de bajo costo. Debido a su bajo costo, después de que estos materiales han sido gastados, estos pueden ser colocados sin una regeneración costosa.

El costo es un importante parámetro para comparar materiales adsorbentes. Sin embargo, la información del costo es pocas veces reportada y el costo de los adsorbentes depende del grado de procesamiento y de la disponibilidad local.

\section{Agradecimientos}

Los autores agradecen a Colciencias y al Departamento de Química de la Universidad del Cauca por el apoyo en la realización de este trabajo. 


\section{Referencias}

[1] S. Bose-O'Reilly, G. Drasch, C. Beinhoff, A. Tesha, K. Drasch, G. Roider, H. Taylor, D. Appleton, U. Siebert, "Health assessment of artisanal gold miners in Tanzania," Sci Total Environ, vol. 408, no.4, pp.796-805, 2010.

[2] Corporación Autónoma Regional de Cundinamarca -CAR. Legalización Ambiental de las Industrias del Curtido de Pieles en Villapinzón, Choconta y Cogua. Bogotá: CAR, 2002.

[3] Ideam, "El impacto ambiental del sector industrial en Colombia," Bogotá: IDEAM. 1998.

[4] Contaminación por mercurio y otros, Distrito minero de Buenos Aires, Cauca. (CRC, Corporación Autónoma Regional del Cauca), "Apoyo a proyectos de producción más limpia en minería para los distritos mineros del Cauca," 2007.

[5] J. Arroyo, D. Gómez, J. Muñoz, "Evolución, impacto y retos del Sector Minero: Un análisis para el Valle del Cauca," Gobernación Valle del Cauca. no.7, pp.3-23, 2013.

[6] Mapa de Dagua-Valle. Disponible en: https://dagua.es.tl/QUEREMAL.htm. Consultado: 20/06/2019.

[7] Mapa rio Cauca y zona del cerrito Valle. Disponible en: https://cvc.gov.co. Consultado: 21/06/2019.

[8] C. Gischler, "Pathways of heavy metals and implications for stakeholders," Sonso lagoon, Colombia, Master Thesis, 2005.

[9] Corporación Autónoma Regional del Cauca (CRC), "Evaluación minero Ambiental deldistritominerodeSuarez," Disponible: http://www.crc.gov.co/files/ConocimientoAmbiental/ mineria/Mineria\%20Suarez/Mineralizacion\%20Suarez.pdf. (Consultada: 04/02/2019), 2006.

[10] L. Baena, "Informe de Evaluación de metales pesados en los sedimentos del canal Navarro," (Canal interceptor Sur), CVC. 2005.

[11] M.A. Barakat, "New trends in removing heavy metals from industral wastewater," Arabian Journal of Chemistry, vol. 4, pp.361-377, 2011.

[12] S. Babel, T.A. Kurniawan, "Low-cost adsorbents for heavy metals uptake from contaminated water: a review," J. Hazard. Mater, vol. 97, pp.219-243, 2003.

[13] F. Fu, Q.Wang, "Removal of heavy metal ions from wastewaters: A review," Journal of Environmental Management, vol. 92, pp.407-418, 2011.

[14] B. Volesky, "Detoxification of metal-bearing efluents: biosorption for the next century. Hydrometallurgy," no.59, pp. 203-216, 2001.

[15] L.K. Wang, D.A. Vaccari, Y.T Hung, N.K. Shamans, "Chemicalprecipitation," En: L.K. Wang, Y.T. Hung, N.K. Shamans, (Eds.), Physicochemical Treatment Processes, vol 3, Humana Press, New Jersey, pp. 141-198, 2004.

[16] H.A. Aziz, M.N. Adlan, K.S. Ariffin, "Heavy metals (Cd, Pb, Zn, Ni, Cu and Cr(III)) removal from water in Malaysia: post treatment by high quality limestone," Bioresour.Technol, vol 99, pp. 1578-1583, 2008.

[17] P.W. Landford, "Toxicity Reduction in Industrial Effluents," Van Nostrand, New York. USA. 1990.

[18] R.W. Gaikwad, V.S. Sapkal, and R.S. Sapkal, "Ion exchange system design for removal of heavy metals from acid mine drainage wastewater," Acta Montanistica Slovaca Ročník, Vol.15, no 4, pp. 298-304, 2010.

[19] M.A. Barakat, "Adsorption behavior of copper and cyanide ions at TiO2-solution interface," J. Colloid Interface Sci, vol. 291, pp. 345-352, 2005.

[20] M.A. Barakat, Y.T. Chen, C.P. Huang, "Removal of toxic cyanide and Cu (II) ions from water by illuminated $\mathrm{TiO}_{2}$ catalyst. J. Appl. Catal," B: Environ, vol.53, pp. 13-20, 2004.

[21] T.Y. Lee, J.W. Park, J.H. Lee, "Waste green sands as a reactive media for the removal of zinc from water," Chemosphere, vol.56, pp. 571-581, 2004.

[22] V.K. Gupta, C.K. Jain, I. Ali, M. Sharma, S.K. Saini, "Removal of cadmium and nickel from wastewater using bagasse fly ash - a sugar industry waste," Water Res. 37, pp. 4038-4044, 2003.

[23] M. Uysal, I. Ar, "Removal of Cr (VI) from industrial wastewaters by adsorption," Part I: determination of optimum conditions. J. Hazard. Mater.149, pp. 482-491, 2007.

[24] U.C. Ghosh, M. Dasgupta, S. Debnath, S.C. Bhat, "Studies on management of chromium(VI)-contaminated industrial waste effluent using hydrous titanium oxide (HTO)," Water, Air, Soil Pollut. 143, pp. 245-256, 2003.

[25] J. Ramírez, Ó. Martinez, L. Fernández, "Remoción de contaminantes en aguas residuales industriales empleando carbón activado de Pino pátula," Avances Investigación en Ingeniería, vol. 10, no. 1, pp. 42-49, 2013.

[26] J.C. Igwe, D.N.Ogunewe, A.A. Abia, "Competitive adsorption of $\mathrm{Zn}(\mathrm{II}), \mathrm{Cd}(\mathrm{II})$ and $\mathrm{Pb}(\mathrm{II})$ ions from aqueous and non-aqueous solution by maize cob and husk. Afr. J. Biotechnol," vol.4, no.10, pp. 1113-1116, 2005.

[27] N.R. Bishnoi, M. Bajaj, N. Sharma, A. Gupta, "Adsorption of Cr(VI) on activated rice husk carbon and activated alumina," Bioresour. Technol., vol. 91, no. 3, pp. 305-307, 2003.

[28] P. Tang, C.K. Lee, K.S. Low, Z. Zainal, "Sorption of $\mathrm{Cr}(\mathrm{VI})$ and $\mathrm{Cu}(\mathrm{II})$ in aqueous solution by 
ethylenediamine modified rice hull," Environ. Technol, vol. 24, pp. 1243-1251, 2003.

[29] I. Ghodbane, and O. Hamdaoui, "Removal of Mercury(II) from aqueous media using eucalyptus bark: Kinetic and equilibrium studies," Journal of Hazardous Materials, vol.160, pp. 301-309, 2008.

[30] Proexport Colombia, "Inversión en el sector Forestal en Colombia," 2012.

[31] R. Sarria-Villa, J. Gallo-Corredor, R. Benítez, M. Páez, V. Campo, "Extraction of phenolic compounds from Pinus patula bark using ethanol-water mixtures and the anti-inflammatory action of the ethanolic extract," International Journal of Current Research. Vol.10, no.03, pp. 66886-66895, 2018.

[32] G. Palma, J. Freerb, J. Baezab, "Removal of metal ions by modified Pinus radiata bark and tannins from water solutions," Water Research, vol.37, pp. 4974-4980, 2003.

[33] G. Vázquez, J. González-Álvarez, S. Freire, M.López-Lorenzo, G. Antorrena, "Removal of cadmium and mercury ions from aqueous solution by sorption on treated Pinus pinaster bark: kinetics and isotherms, Bioresource Technology," vol. 82, no. 3, pp.247-251, 2002.

[34] J. Beltrán-Heredia, J. Sánchez-Martín, M.C. Gómez-Muñoz, "New coagulant agents from tannin extract: preliminary optimization studies," Chem. Eng. J, vol.162, pp.1019-1025, 2010.

[35] K. Kesenci, R. Say, A. Denizli, "Removal of heavy metal ions from water by using poly(ethyleneglycoldimethacrylate-co-acrylamide) beads," Eur. Polym. J., vol. 38, pp. 1443-1448, 2002.

[36] S. Vigneswaran, H.H. Ngo, D.S. Chaudhary, Y.T. Hung, "Physico-chemical treatment processes for water reuse," Wang, L.K; Hung, Y.T; Shammas, N.K (Eds.), Physicochemical Treatment Processes, Humana Press, New Jersey, vol. 3, pp. 635-676, 2004.

[37] N. Saffaj, H. Loukil, S.A. Younssi, A. Albizane, M. Bouhria, M. Persin, A. Larbot, "Filtration of solution containing heavy metals and dyes by means of ultrafiltration membranes deposited on support made of Morrocan clay," Desalination, vol.168, pp. 301-306, 2004.

[38] T.A. Kurniawan, Y.S. Chan, W.H. Lo, S. Babel, "Physico-chemical treatment techniques for wastewater laden with heavy metals," Chem. Eng. J, vol.118, pp. 83-98, 2006.

[39] W.P. Zhu, J. Gao, S.P. Sun, S. Zhang, T.S. Chung, "Poly(amidoamine) dendrimer (PAMAM) grafted on thin film composite (TFC) nanofiltration (NF) hollow fiber membranes for heavy metal removal," Journal of Membrane Science, vol. 487, no.1, pp. 117-126, 2015.

[40] H. A. Qdais, and H. Moussa, "Removal of heavy metals from wastewater by membrane processes: A comparative study," Desalination, vol.164, no.2, pp.105-110, 2004.

[41] M.R. Jakobsen, J.F. Rasmussen, S. Nielsen, L.M. Ottosen," Electrodialytic removal of cadmium from wastewater sludge," J. Hazard. Mater., vol.106B, pp. 127-132, 2004.

[42] T. Mohammadi, A. Razmi, M. Sadrzadeh, "Effect of operatingparameters on $\mathrm{Pb}^{2+}$ separation from wastewater using electrodialysis," Desalination, vol.167, pp. 379-385, 2004.

[43] J.Wu, H. Zhang, P.J. He, Q. Yao, L.M. Shao, "Cr (VI) removal from aqueous solution by dried activated sludge biomass," Journal of hazardous materials. Vol.176, no.1, pp. 697-703, 2010.

[44] J.M. Herrmann, "Heterogeneous photocatalysis: fundamentals and applications to the removal of various types of aqueous pollutants," Catal. Today, vol. 53, pp. 115-129, 1999.

[45] P. Kajitvichyanukula, J. Ananpattarachaia, S. Pongpom, "Sol-gel preparation and properties study of $\mathrm{TiO}_{2}$ thin film for photocatalytic reduction of chromium(VI) in photocatalysis process," Sci. Technol. Adv. Mater., vol. 6, pp. 352-358, 2005.

[46] E. Gkika, A. Troupis, A. Hiskia, E. Papaconstantinou, "Photocatalytic reduction of chromium and oxidation of organics by polyoxometalates.Appl," Catal. B: Environ., vol.62, pp. 28-34, 2006.

[47] A. Aklil, M. Mouflihb, S. Sebti, "Removal of heavy metal ions from water by using calcined phosphate as a new adsorbent. J. Hazard. Mater," A vol.112, pp. 183-190, 2004.

[48] T. A. Mohammadi, A. Mohebb, M. Sadrzadeh, A. Razmi, "Modeling of metal ion removal from wastewater by electrodialysis," Sep. Purif. Technol, vol. 41, pp. 73-82, 2005.

[49] S.E. Pabón, R. Benítez, R.A. Sarria-Villa y J.A. Gallo, "Contaminación del agua por metales pesados, métodos de análisis y tecnologías de remoción. Una revisión," Entre Ciencia e Ingeniería, vol. 14(27), pp. 9-18, 2020.
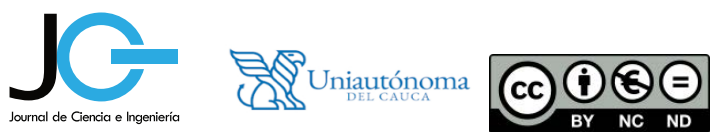